\title{
Prediction of Chatter Stability for Milling Process Using an Improved Semi-discretization Method
}

\author{
LI Zhongqun ${ }^{1, a}$, ZHU Fan ${ }^{1, b}$, XIA Lei $^{1, b}$, PENG Yuerong ${ }^{1, c}$ \\ ${ }^{1}$ School of Mechanical Engineering, Hunan University of Technology, Zhuzhou, 412007, China \\ azhqunli@163.com, ${ }^{\text {b } 522657039 @ q q . c o m ~, ~}{ }^{\text {c3 } 343782710 @ q q . c o m, ~ d 872976898 @ q q . c o m ~}$
}

Keywords: Milling Process; Stability Lobe Diagram; Anaytical Solution; Semi-discretization Method; Abstract. On the basis of the classical semi-disretization method (SDM) proposed by Insperger, an improved SDM and its algorithm were implemented in this paper. With the developed module, the chatter stability of milling process can be predicted. As the dichotomy search rather than sequential search is applied, the simulation time is greatly reduced. As a result, it is pratical to determine the optimized chatter-free cutting conditions of milling process in shop floor applications.

\section{Introduction}

In milling process, chatter has negative effect on the machined surface quality, the cutting tool and even the machine tool. Chatter stability prediction is an important and effective way for optimal selection of spindle speed and cutting depth to avoid chatter and improve production efficiency. Based on the classical dynamic model considering the regenerative effect described by a Delay Diffential Equation (DDE) with time varying coefficents [1], the stability lobe diagrams (SLDs) can be obtained to find the relations between the cutting depths and the spindle speeds [2,3]. It contains stability boundaries, below which vibration dies down and above which vibration grows to finite amplitudes eventually.

Except experimental methods [4], numerical algorithms of predicting stability have been developed. The basic way is to tranform DDEs from time domain to frequency domain using the Laplace Transform. Then to calculate the ciritcal axial cutting depth utilizing real and image part of the characteristic equation of the system. Altintas [5] presented an analytical method for predicting milling stability based on the mean of the Fourier series of the dynamic milling coefficients.

ZOA (Zero-order Analytical) method is efficient and fast, but it cannot predict the existence of the additional stability regions in low immersion milling. To overcome the problem, Multi-frequency Solution (MFS), Semi-discretization Method (SDM) and Full-discretization Method (FDM) have been developed. The MFS was first explored by Budak [6] and then extended by Merdol [7]. The SDM was developed by Insperger [8], and it is efficient in stability analysis of linear delayed systems which can be applied to predict milling stability. Ding [9] presented the FDM to predict of milling stability which has high computational efficiency without loss of any numerical precision compared with the SDM.

As the effect of high harmonics due to highly interrupted cutting was considered, the computation efficiency of SDM is not high. Moreover, to obtain a SLD with SDM, the core program must be looped for both discretized spindle speeds and available cutting depths, it is very time consuming. Therefore, a dichotomy search based SDM is proposed in the paper to predict the chatter stability for milling operation.

\section{Stability analysis of 2 DOF milling process with SDM}

The governing equation of a 2-DOF milling model with a couple and delay differential equation reads 


$$
\left\{\begin{array}{l}
\zeta_{x} \omega_{n x}(t)+\omega_{n x}^{2} x(t) \\
\zeta_{y} \omega_{n y}(t)+\omega_{n y}^{2} y(t)
\end{array}\right\}=\frac{1}{2} a_{\mathrm{p}} K_{\mathrm{t}}\left[\begin{array}{cc}
\frac{\omega_{n x}^{2}}{k_{x}} & 0 \\
0 & \frac{\omega_{n y}^{2}}{k_{y}}
\end{array}\right]\left[\begin{array}{ll}
a_{x x}(t) & a_{x y}(t) \\
a_{y x}(t) & a_{y y}(t)
\end{array}\right]\left\{\begin{array}{l}
x(t)-x(t-T) \\
y(t)-y(t-T)
\end{array}\right\}
$$

where $\omega_{\mathrm{nx}}, \zeta_{\mathrm{x}}, k_{\mathrm{x}}, \omega_{\mathrm{ny}}, \zeta_{\mathrm{y}}, k_{\mathrm{y}}$ are the natural frequency, relative damping and stiffness in $x$ and $y$ directions; $a_{\mathrm{p}}$ is the axial cutting depth; $K_{\mathrm{t}}$ is the tangential specific cutting force; $T$ is tooth passing peroid; $x(t-T)$ and $y(t-T)$ are the delayed term; the time varying directional coefficients reads

$$
\begin{aligned}
& a_{x x}(t)=\sum_{j=1}^{N}-g_{j}(t)\left[\sin 2 \phi_{j}(t)+K_{\mathrm{r}}\left(1-\cos 2 \phi_{j}(t)\right)\right] \\
& a_{x y}(t)=\sum_{j=1}^{N}-g_{j}(t)\left[\left(1+\cos 2 \phi_{j}(t)\right)+K_{\mathrm{r}} \sin 2 \phi_{j}(t)\right] \\
& a_{y x}(t)=\sum_{j=1}^{N} g_{j}(t)\left[\left(1-\cos 2 \phi_{j}(t)\right)+K_{\mathrm{r}} \sin 2 \phi_{j}(t)\right] \\
& a_{y y}(t)=\sum_{j=1}^{N} g_{j}(t)\left[\sin 2 \phi_{j}(t)-K_{\mathrm{r}}\left(1+\cos 2 \phi_{j}(t)\right)\right]
\end{aligned}
$$

where $N$ is the number of the teeth, $K_{\mathrm{r}}$ is the ratio of radial specific cutting force with respect of tangential specific cutting force, $\phi_{\mathrm{j}}(\mathrm{t})$ is angular position of tooth $j$ defined as

$$
\phi_{j}(t)=(2 \pi \Omega / 60) t+(j-1) \pi / N
$$

where $\Omega$ is the spindle speed in $\mathrm{r} / \mathrm{min}$. The function $g(\phi(t))$ is a screen function, if the tooth $j$ is in cut, it is equal 1 ; otherwise, it is equal to 0

In the $i$ th semi-discretization interval, Equation (1) can be approximated as

$$
\left\{\begin{array}{l}
\left\{(t)+2 \zeta_{x} \omega_{n x}(t)+\omega_{n x}^{2} x(t)\right. \\
\zeta_{y} \omega_{n y}(t)+\omega_{n y}^{2} y(t)
\end{array}\right\}=\frac{1}{2} a_{\mathrm{p}} K_{\mathrm{t}}\left[\begin{array}{cc}
\frac{\omega_{n x}^{2}}{k_{x}} & 0 \\
0 & \frac{\omega_{n y}^{2}}{k_{y}}
\end{array}\right]\left[\begin{array}{ll}
\alpha_{x x i} & \alpha_{x y i} \\
\alpha_{y x i} & \alpha_{y y i}
\end{array}\right]\left\{\begin{array}{l}
x(t)-x_{\tau, i} \\
y(t)-y_{\tau, i}
\end{array}\right\}
$$

where the average direction coeffiency reads

$$
\alpha_{i}=\frac{1}{\Delta t} \int_{t_{i}}^{t_{i+1}} a(t) \mathrm{d} t
$$

By Cauchy transformation, Equation (5) can be written in the following form

$$
\phi(t)=\mathbf{A}_{i} \mathbf{q}(t)+\frac{1}{2} \mathbf{B}_{i} \mathbf{q}_{i-m+1}+\frac{1}{2} \mathbf{B}_{i} \mathbf{q}_{i-m}
$$

where 


$$
\begin{aligned}
& \mathbf{A}_{i}=\left[\begin{array}{ccccc}
0 & 0 & 1 & 0 \\
0 & 0 & 0 & 1 \\
-\omega_{n x}^{2}-\frac{a_{\mathrm{p}} \omega_{n x}^{2} h_{x x i}}{k_{x}} & -\frac{a_{\mathrm{p}} \omega_{n x}^{2} h_{x y i}}{k_{x}} & -2 \zeta_{x} \omega_{n x} & 0 \\
-\frac{a_{\mathrm{p}} \omega_{n y}^{2} h_{y x i}}{k_{y}} & -\omega_{n y}^{2}-\frac{a_{\mathrm{p}} \omega_{n y}^{2} h_{y y}}{k_{y}} & 0 & -2 \zeta_{y} \omega_{n y}
\end{array}\right], \mathbf{B}_{i}=\left[\begin{array}{cccc}
0 & 0 & 0 & 0 \\
0 & 0 & 0 & 0 \\
\frac{a_{\mathrm{p}} \omega_{n x}^{2} h_{x x i}}{k_{x}} & \frac{a_{\mathrm{p}} \omega_{n x}^{2} h_{x y i}}{k_{x}} & 0 & 0 \\
\frac{a_{\mathrm{p}} \omega_{n y}^{2} h_{y x i}}{k_{y}} & \frac{a_{\mathrm{p}} \omega_{n y}^{2} h_{y y i}}{k_{y}} & 0 & 0
\end{array}\right] \\
& \mathbf{u}(t)=(x(t), y(t), \delta(t), x(t))^{\prime} \text { and } \mathbf{u}_{j}=\mathbf{u}\left(t_{j}\right)=\left(x\left(t_{j}\right), y\left(t_{j}\right), \delta\left(t_{j}\right), x\left(t_{j}\right)\right)^{\prime}
\end{aligned}
$$

Then the solution Equation (9) for the initial condition $\mathbf{u}\left(t_{i}\right)=\mathbf{u}_{i}$ reads

$$
\mathbf{u}_{i+1}=\mathbf{P}_{i} \mathbf{u}_{i}+\mathbf{R}_{i}\left(\mathbf{u}_{i-m+1}+\mathbf{u}_{i-m}\right) / 2
$$

where

$$
\mathbf{P}_{i}=\exp \left(\mathbf{A}_{i} \Delta t\right) \quad \mathbf{R}_{i}=\left(\exp \left(\mathbf{A}_{i} \Delta t\right)-\mathbf{I}\right) \mathbf{A}_{i}^{-1} \mathbf{B}_{i}
$$

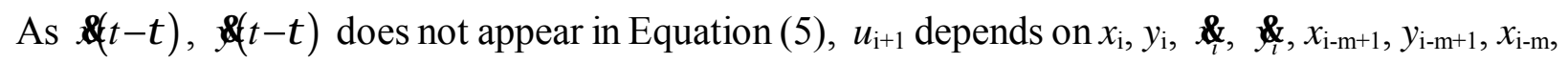

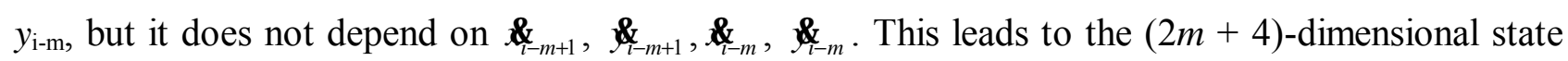
vector

$$
\mathbf{z}_{i}=\operatorname{col}\left(x_{i}, y_{i}, \underset{i}{\&}, x_{i-1}, y_{i-1}, \mathrm{~L}, x_{i-m}, y_{i-m}\right)
$$

The resulting discrete map reads

$$
\mathbf{z}_{i+1}=\mathbf{D}_{i} \mathbf{z}_{i}
$$

Where the $(2 m+4)$-dimensional coefficient matrix is

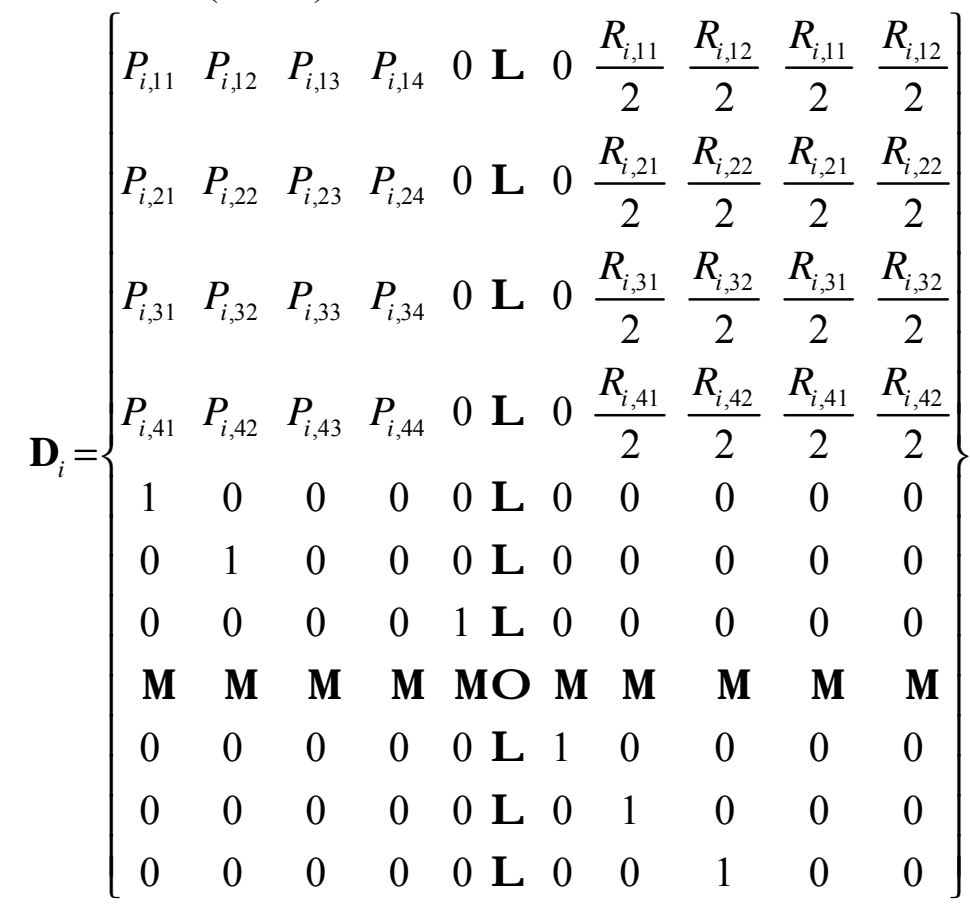


Where $P_{i, h j}$ and $R_{i, h j}$ are the elements of matrices $\mathrm{P}_{i}$ and $\mathrm{R}_{i}$ in the $h$ th row and $j$ th column. The $(2 m+4)$-dimensional transition matrix $\Phi$ is determined by coupling Equation (15) for $i=0,1, \ldots, k-1$ :

$$
\Phi=\mathbf{D}_{k-1} \mathbf{D}_{k-2} \mathrm{~L} \quad \mathbf{D}_{2} \mathbf{D}_{1}
$$

If the eigenvalues of $\Phi$ are in modulus less than one, then the system is stable.

\section{Implementation of stability lobe diagram with SDM}

Based on the proposed SDM algorithm for stability analysis of 2-DOF milling system, a Matlab-based simulation module has been developed, which reads the input data such as the modal parameter, tool geometry, specific cutting forces and cutting conditions. The flow chart is shown in Fig.1. It can be found that two improvements have been made with regard to the original program provided by Insperger [11]. Firstly, an iterative dichotomy search instead of a sequential search was used to determine the stable borders which results in a decreased calculation amount by an order of magnitude. Secondly, a one-dimensional array instead of a two-dimensional array was used to store the SLD which results in a decreased of memory space. Thus, the simulation time is greatly reduced.

\section{Simulation and analysis}

SLD are determined for down milling where the radial depth of cut $a_{\mathrm{e}}$ is set as $1.0,0.5,0.1,0.05$ times tool diameter $D$ with a two-fluted cutter. The modal parameters of dominant mode of the milling system are $\omega_{\mathrm{nx}}=900 \mathrm{~Hz}, \zeta_{\mathrm{x}}=0.01, k_{\mathrm{x}}=5 \times 10^{6} \mathrm{~N} / \mathrm{m} ; \omega_{\mathrm{ny}}=900 \mathrm{~Hz}, \zeta_{\mathrm{y}}=0.01, k_{\mathrm{y}}=5 \times 10^{6} \mathrm{~N} / \mathrm{m}$. The tangential and radial specifc cutting forces are $K_{\mathrm{t}}=7.96 \times 10^{8} \mathrm{~N} / \mathrm{m}^{2}$ and $K_{\mathrm{r}}=1.6810^{8} \mathrm{~N} / \mathrm{m}^{2}$. The SLDs for various $a_{\mathrm{e}}$ are shown in Fig. 2. It is shown that when $a_{\mathrm{e}}$ is very low, i.e. the process is highly intermittent, the SLDs are more accurately predicted by using SDM than by ZOA (see Fig. 3). The accuracy of the SDM is limited only by its sampling interval and the computation time depends on the number of modes in the system and sampling interval. The approximatin parameter for the SDM is $m=$ 40, the same as Refrences [11]. Upon the same $2.4 \mathrm{GHz}$ Intel ${ }^{\circledR}$ Core (TM) 2 Duo CPU, the computation time for determining SLD was $110 \mathrm{~s}$ using the improved algorithm vs $243 \mathrm{~s}$ using the Insperger's original program. It is shown that with the improved algorithm, the total computation time was reduced greatly, and thus makes it possible to apply it in shop-floor application. 


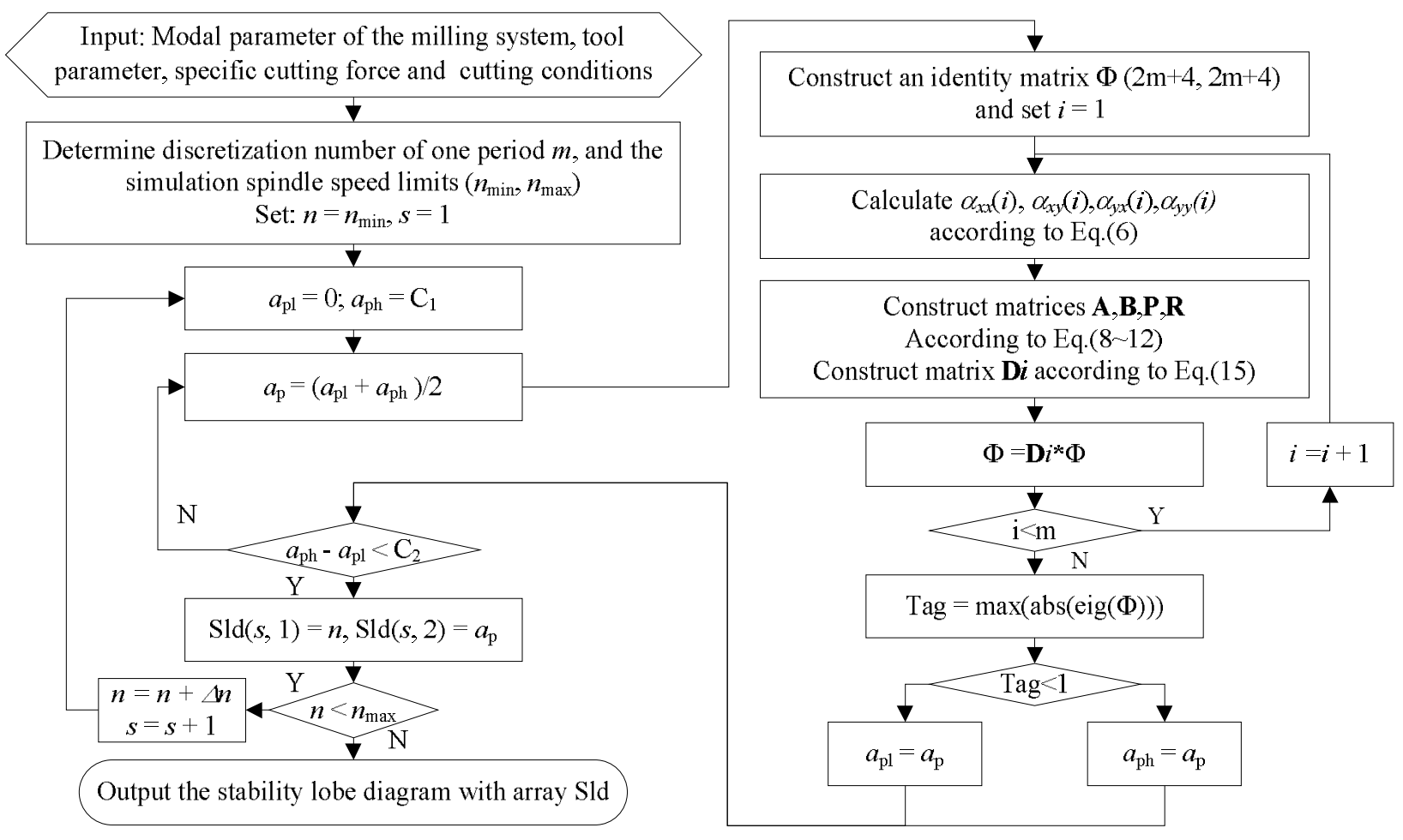

Fig.1 Flow chart of obtaining stability lobe diagram for milling process with SDM

\section{Conclusion}

An improved SDM algorithm was used to predict the SLDs of milling process in this paper. On one hand, as it considers the time variation of directional factors at each discrete sampling interval, it can predict the stability lobes at any speed and have better prediction accuracy than ZOA, especially when the radial depth of cut is very small. On the other hand, as dichotomy search other than sequential search was used to calculate the critical cutting depth, both the cycles and the computing time have been reduced greatly, and thus make it possible to be applied in engineering applications.

\section{Acknowledgement}

This work was financially spported by the National Natural Science Foundation of China (51375160, 51375161), National Major Science and Technology Special Projects of China (2102ZX04011-011).

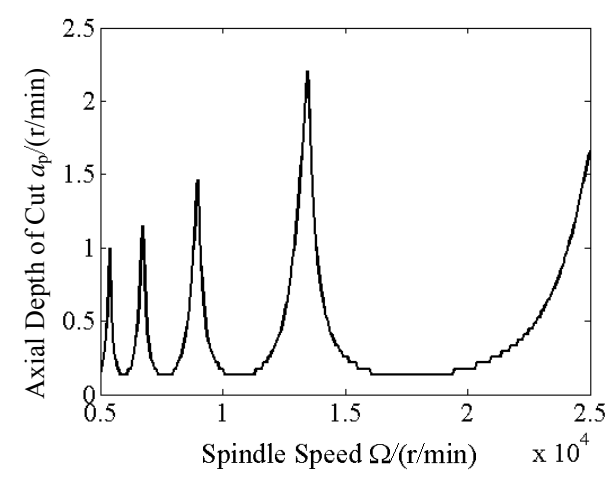

(a) $a_{\mathrm{e}} / D=1$

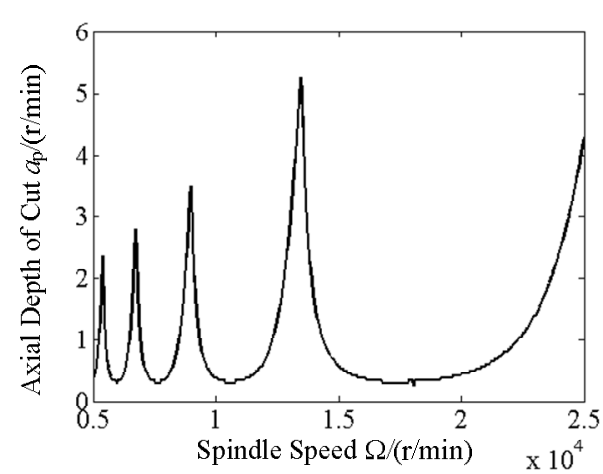

(b) $a_{\mathrm{e}} / D=0.5$ 


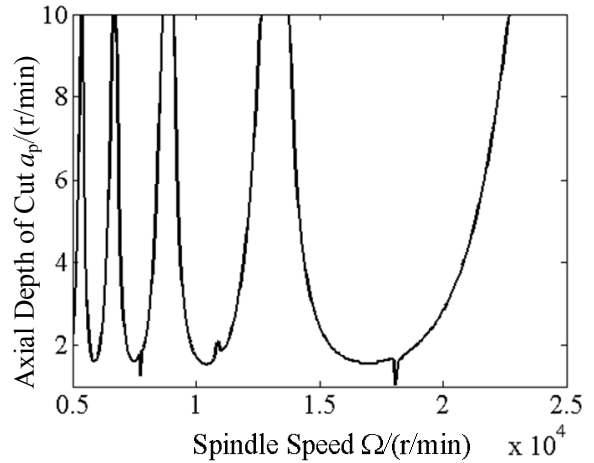

(c) $a_{\mathrm{e}} / D=0.1$

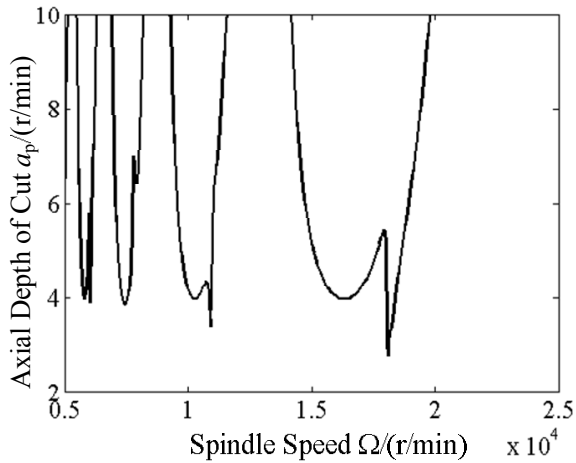

(d) $a_{\mathrm{e}} / D=0.05$

Fig.2 Stability lobe diagram for 2 DOF down milling with different radial depth of cut

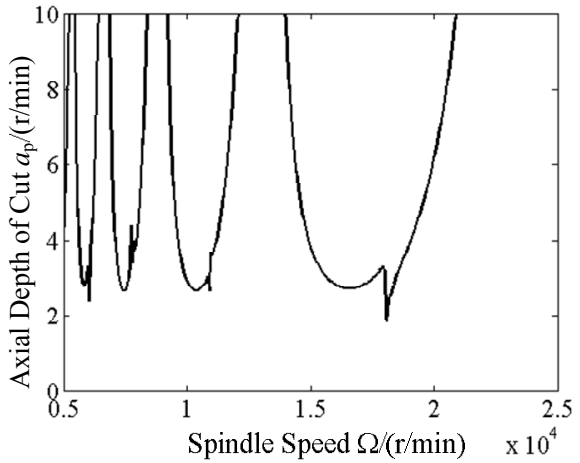

(a) SDM

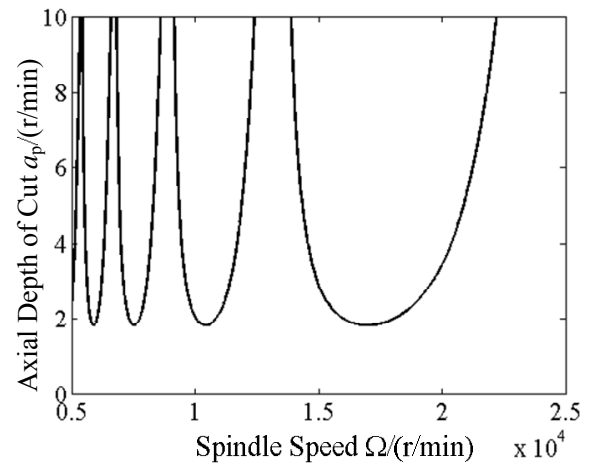

(b) ZOA

Fig.3 Comparison of stability lobe diagrams obtained with different method $\left(a_{\mathrm{e}}=0.1 D\right)$

\section{References}

[1] R. Sridhar, R.E. Hohn and G.W. Long: J. Eng. Ind. Vol. 90(1968): p. 330

[2] S.A. Tobias: Machine tool vibrations (Blackie and Sons Ltd., Glasgow 1965)

[3] Y. Altintas and E. Budak: Annals of the CIRP, Vol. 44(1995), p. 357

[4] F. Ismail and E. Soliman: Int J. Mach. Tool Manu. Vol.37 (1997): p. 763

[5] Y. Altintas and E. Budak: CIRP Annals - Manuf. Techn. Vol. 44(1995): p. 357

[6] E. Budak and Y. Altintas: J. Dyn. Syst-T ASME Vol. 120(1998): p. 22

[7] S.D. Merdol and Y.Altintas: J. Manuf. Sci. Eng. Vol. 126(2004): p. 459

[8] T. Insperger and G. Stepan: Int. Numer. Meth. Eng, Vol. 55 (2002): 503-518.

[9] Y. Ding, L.M. Zhu, X. J. Zhang and H. Ding: Int J. Mach. Tool Manu. Vol. 50(2010): p.502 